\title{
Finite Element Simulation of Heat Transfer in Ferrofluid
}

\author{
Tomasz Strek \\ Poznan University of Technology, Institute of Applied Mechanics \\ Poland
}

\section{Introduction}

During the last decades, an extensive research work has been done on the fluids dynamics in the presence of magnetic field (magnetorheological fluids and ferrofluids). The effect of magnetic field on fluids is worth investigating due to its innumerable applications in wide spectrum of fields. The study of interaction of the magnetic field or the electromagnetic field with fluids have been documented e.g. among nuclear fusion, chemical engineering, medicine, high speed noiseless printing and transformer cooling.

Ferrofluids are industrially prepared magnetic fluids which consist of stable colloidal suspensions of small single-domain ferromagnetic particles in suitable carrier liquids. Usually these fluids do not conduct electric current and exhibit a nonlinear paramagnetic behavior.

An external magnetic field imposed on a ferrofluid with varying susceptibility, e.g., due to a temperature gradient, results in a non-uniform magnetic body force, which leads to a form of heat transfer called thermomagnetic convection. This form of heat transfer can be useful when conventional convection heat transfer is inadequate, e.g., in miniature microscale devices or under reduced gravity conditions.

This chapter contains numerical results of computer simulation of heat transfer through a ferrofluid in channel flow under the influence of magnetic dipole, ferrofluid cooling of heatgenerating device and heat transfer through a ferrofluid in channel with porous walls.

All considered problems describe two-dimensional time dependent heat transfer through a ferrofluid in channel in response to magnetic force arising from the magnetic field of the magnetic dipole. Numerical results show us the potential of ferrofluid in cooling of heatgenerating devices. In last problem we can observe a form of heat transfer called thermomagnetic convection.

In this chapter results of time dependent problems are obtained using standard computational fluid dynamics code COMSOL Multiphyisics with modifications to account for the magnetic term (e.g. the Kelvin body force and the thermal power) when needed. COMSOL is a powerful interactive environment for equation-based multiphysics modeling and solving all kinds of scientific and engineering problems based on partial differential equations using the finite element method.

The governing equations for considered problems are derived from conservation of energy, momentum, heat and magnetic induction equation. The resulting equations depend on the properties of ferrofluid and magnetic field. In this chapter magneto-thermo-mechanical 
problems are modeled by coupled dimensionless equations. Governed equations cannot generally be determined without a supplementary assumptions.

\section{Ferrofluids}

Ferrofluids (Rosensweig, 1985) are non-conducting fluids and the study of the effect of magnetisation has yielded interesting information. In equilibrium situation the magnetization property is generally determined by the fluid temperature, density and magnetic field intensity and various equations, describing the dependence of static magnetization on these quantities. The simplest relation is the linear equation of state. It can be assumed that the magneto-thermo-mechanical coupling is not only described by a function of temperature, but by an expression involving also the magnetic field strength. This assumption permit us not to consider the ferrofluid far away from the sheet at Curie temperature in order to have no further magnetization. This feature is essential for physical applications because the Curie temperature is very high (e.g. 1043 Kelvin degrees for iron) and such a temperature would be meaningless for applications concerning most of ferrofluids. So instead of having zero magnetization far away from the sheet, due to the increase of fluid temperature up to the Curie temperature this formulation allows us to consider whatever temperature is desired and the magnetisation will be zero due to the absence of the magnetic field sufficiently far away from the sheet.

Moreover, ferrofluids are mostly organic solvent carriers having ferromagnetic oxides, acting as solute. Ferrofluids consist of colloidal suspensions of single domain magnetic nanoparticles. They have promising potential for heat transfer applications, since a ferrofluid flow can be controlled by using an external magnetic field (Ganguly et al., 2004). However, the relationship between an imposed magnetic field, the resulting ferrofluid flow and the temperature distribution is not understood well enough. The literature regarding heat transfer with magnetic fluids is relatively sparse.

An overview of prior research on heat transfer in ferrofluid flows e.g. thermomagnetic free convection, thermomagnetic forced convection and boiling, condensation and multiphase flow are presented in paper (Ganguly et al., 2004). Many researchers are seeking new technologies to improve the operation of existing oil-cooled electromagnetic equipment. One approach suggested in literature is to replace the oil in such devices with oil-based ferrofluids, which can take advantage of the pre-existing leakage magnetic fields to enhance heat transfer processes. In paper (Tangthieng et al., 1999) authors present results of an initial study of the enhancement of heat transfer in ferrofluids in magnetic fields which are steady but variable in space. Finite element simulations of heat transfer to a ferrofluid in the presence of a magnetic field are presented for flow between flat plates and in a box. The natural convection of a magnetic fluid in a partitioned rectangular cavity was considered in paper (Yamaguchi et al., 2002). It was found that the convection state may be largely affected by improving heat transfer characteristic at higher Rayleigh number when a strong magnetic field was imposed. Numerical results of combined natural and magnetic convective heat transfer through a ferrofluid in a cube enclosure were presented in paper (Snyder et al., 2003). The purpose of this work was to validate the theory of magnetoconvection. The magnetoconvection is induced by the presence of magnetic field gradient. The Curie law states that magnetization is inversely proportional to temperature. That is way the cooler ferrofluid flows in the direction of the magnetic field gradient and displaces hotter ferrofluid. This effect is similar to natural convection where cooler, more 
dense, material flows towards the source of gravitational force. Results were obtained using standard computational fluid dynamics codes with finite element method.

The effect of magnetic field on the viscosity of ferroconvection in an anisotropic porous medium was studied in paper (Ramanathan \& Suresh, 2004). It was found that the presence of anisotropic porous medium destabilizes the system, where as the effect of magnetic field dependent viscosity stabilizes the system. In this paper the investigated fluid was assumed to be incompressible having variable viscosity. Experimentally it has been demonstrated in prior research that the magneto viscosity has got exponential variation, with respect to magnetic field. As a first approximation for small field variation, linear variation of magneto viscosity has been used in paper (Ramanathan \& Suresh, 2004).

One of the problems associated with drug administration is the inability to target a specific area of the body. Among the proposed techniques for delivering drugs to specific locations within the human body, magnetic drug targeting (Voltairas et al., 2002, Ritter et al., 2004) surpasses due to its non-invasive character and its high targeting efficiency. Although the method has been proposed almost 30 years ago, the technical problems obstruct possible applications. It was the aim of the paper (Voltairas et al., 2002) to classify the emerging problems and propose satisfactory answers. A general phenomenological theory was developed and a model case was studied, which incorporates all the physical parameters of the problem. A hypothetical magnetic drug targeting system, utilizing high gradient magnetic separation principles, was studied theoretically using FEMLAB simulations in paper (Ritter et al., 2004). This new approach uses a ferromagnetic wire placed at a bifurcation point inside a blood vessel and an externally applied magnetic field, to magnetically guide magnetic drug carrier particles through the circulatory system and then to magnetically retain them at a target site.

\section{Governing equations}

\subsection{The magnetic field intensity}

In this paper the considered flow is influenced by magnetic dipole. We assumed that the magnetic dipole is located at distance $|b|$ below the sheet at point $(a, b)$. The magnetic dipole gives rise to a magnetic field, sufficiently strong to saturate the fluid. In the magnetostatic case where there are no currents present, Maxwell-Ampere's law reduces to $\nabla \times \mathbf{H}=\mathbf{0}$. When this holds, it is also possible to define a magnetic scalar potential by the relation $\mathbf{H}=-\nabla V_{m}$ and its scalar potential for the magnetic dipole is given by

$$
V_{m}(\mathbf{x})=V_{m}\left(x_{1}, x_{2}\right)=\frac{\gamma}{2 \Pi} \frac{x_{1}-a}{\left(x_{1}-a\right)^{2}+\left(x_{2}-b\right)^{2}} .
$$

where $\gamma$ is the magnetic field strength at the source (of the wire) and $(a, b)$ is the position were the source is located.

\subsection{Heat transfer and fluid flow}

The governing equations of the fluid flow under the action of the applied magnetic field and gravity field are: the mass conservation equation, the fluid momentum equation and the energy equation for temperature in the frame of Boussinesque approximation. 
The mass conservation equation for an incompressible fluid is

$$
\nabla \cdot \mathbf{v}=0 .
$$

The momentum equation for magnetoconvective flow is modified from typical natural convection equation by addition of a magnetic term

$$
\rho_{0}\left(\frac{\partial \mathbf{v}}{\partial t}+\mathbf{v} \cdot \nabla \mathbf{v}\right)=-\nabla p+\nabla \cdot \mathbf{S}+\alpha \rho_{0} g\left(T-T_{0}\right) \mathbf{k}+(\mathbf{M} \cdot \nabla) \mathbf{B}
$$

where $\rho_{0}$ is the density, $\mathbf{v}$ is the velocity vector, $p$ is the pressure, $T$ is the temperature of the fluid, $\mathbf{S}$ is the extra stress tensor, $\mathbf{k}$ is unit vector of gravity force and $\alpha$ is the thermal expansion coefficient of the fluid.

The energy equation for an incompressible fluid which obeys the modified Fourier's law is

$$
\rho_{0} c\left(\frac{\partial T}{\partial t}+\mathbf{v} \cdot \nabla T\right)=k \nabla^{2} T+\eta \Phi-\mu_{0} T \frac{\partial \mathbf{M}}{\partial T} \cdot((\mathbf{v} \cdot \nabla) \mathbf{H})
$$

where $k$ is the thermal conductivity, $\eta$ is the viscosity and $\eta \Phi$ is the viscous dissipation

$$
\Phi=\left(2\left(\left(\frac{\partial v_{1}}{\partial x}\right)^{2}+\left(\frac{\partial v_{2}}{\partial y}\right)^{2}\right)+\left(\frac{\partial v_{2}}{\partial x}+\frac{\partial v_{1}}{\partial y}\right)^{2}\right)
$$

The last term in the energy equation represents the thermal power per unit volume due to the magnetocaloric effects.

\subsection{The Kelvin body force for magnetoconvective flow}

The last term in the momentum equation represents the Kelvin body force per unit volume

$$
\mathbf{f}=(\mathbf{M} \cdot \nabla) \mathbf{B},
$$

which is the force that a magnetic fluid experiences in a spatially non-uniform magnetic field. We have established the relationship between the magnetization vector and magnetic field vector

$$
\mathbf{M}=\chi_{m} \mathbf{H} .
$$

Using the constitutive relation (relation between magnetic flux density and magnetic field vector) we can write the magnetic induction vector in the form

$$
\mathbf{B}=\mu_{0}\left(1+\chi_{m}\right) \mathbf{H} .
$$

The variation of the total magnetic susceptibility $\chi_{m}$ is treated solely as being dependent on temperature (Ganguly et al., 2004)

$$
\chi_{m}=\chi_{m}(T)=\frac{\chi_{0}}{1+\alpha\left(T-T_{0}\right)} .
$$


Finally, the Kelvin body force can be represented by

$$
\mathbf{f}=\frac{1}{2} \mu_{0} \chi_{m}\left(1+\chi_{m}\right) \nabla(\mathbf{H} \cdot \mathbf{H})+\mu_{0} \chi_{m} \mathbf{H}\left((\mathbf{H} \cdot \nabla) \chi_{m}\right) .
$$

Using equations (7)-(8) we can write Eq. (3) and (4) in the form, respectively

$$
\begin{aligned}
& \rho_{0}\left(\frac{\partial \mathbf{v}}{\partial t}+\mathbf{v} \cdot \nabla \mathbf{v}\right)=-\nabla p+\nabla \cdot \mathbf{S}+\alpha \rho_{0} g\left(T-T_{0}\right) \mathbf{k}+ \\
& +\frac{1}{2} \mu_{0} \chi_{m}\left(1+\chi_{m}\right) \nabla(\mathbf{H} \cdot \mathbf{H})+\mu_{0} \chi_{m} \mathbf{H}\left((\mathbf{H} \cdot \nabla) \chi_{m}\right)
\end{aligned}
$$

and

$$
\rho_{0} c\left(\frac{\partial T}{\partial t}+\mathbf{v} \cdot \nabla T\right)=k \nabla^{2} T+\eta \Phi-\mu_{0} T \frac{\partial\left(\chi_{m} \mathbf{H}\right)}{\partial T} \cdot((\mathbf{v} \cdot \nabla) \mathbf{H})
$$

\subsection{The Brinkman equations for porous media flow}

Fluid and flow problems in porous media have attracted the attention of industrialists, engineers and scientists from varying disciplines, such as chemical, environmental, and mechanical engineering, geothermal physics and food science. There has been a increasing interest in heat and fluid flows through porous media.

The Brinkman equations describe flow in porous media where momentum transport by shear stresses in the fluid is of importance. The model extends Darcy's law to include a term that accounts for the viscous transport, in the momentum balance, and introduces velocities in the spatial directions as dependent variables. The flow field is determined by the solution of the momentum balance equations in combination with the continuity equation

$$
\rho_{0} \frac{\partial \mathbf{v}_{B}}{\partial t}=-\nabla p_{B}+\nabla \cdot \mathbf{S}_{B}+\frac{\eta}{k_{p}} \mathbf{v}_{B}+\mathbf{F}
$$

where $\eta$ is the viscosity, $k_{p}$ is the permeability of the porous structure (unit: $m^{2}$ ).

The Brinkman equations applications are of great use when modelling combinations of porous media and free flow. The coupling of free media flow with porous media flow is common in the field of chemical engineering. This type of problems arises in filtration and separation and in chemical reaction engineering, for example in the modelling of porous catalysts in monolithic reactors.

Flow in the free channel is described by the Navier-Stokes equations and the mass conservation equation described in previous sections. In the porous domain, flow is described by the Brinkman equations according

$$
\rho_{0} \frac{\partial \mathbf{v}_{B}}{\partial t}=-\nabla p_{B}+\nabla \cdot \mathbf{S}_{B}+\frac{\eta}{k_{p}} \mathbf{v}_{B}
$$

and

$$
\nabla \cdot \mathbf{v}_{B}=0
$$




\subsection{The dimensionless equations}

For simplicity the preferred work choice is to work in non-dimensional frame of reference. Now some dimensionless variables will be introduced in order to make the system much easier to study (Strek, 2005, Strek \& Jopek, 2007). Moreover some of the dimensionless ratios can be replaced with well-known parameters: the Prandtl number Pr, the Rayleigh number $R a$, the Eckert number $E c$, the Reynolds number $R e$, the Darcy number $D a$ and the magnetic number $M n$, respectively:

$$
\begin{gathered}
\operatorname{Pr}=\frac{\eta_{0}}{\rho_{0} \kappa}, R a=\frac{\alpha \rho_{0} g h^{3} \delta T}{\eta_{0} \kappa}, E c=\frac{v_{r}^{2}}{c \delta T}=\frac{\kappa^{2}}{c \delta T h^{2}}, R e=\frac{h \rho_{0} v_{r}}{\eta_{0}}=\frac{\rho_{0} \kappa}{\eta_{0}}, D a=\frac{h^{2}}{k_{p}}, \\
M n=\frac{\mu_{0} H_{r}^{2}}{\rho_{0} v_{r}^{2}}=\frac{\mu_{0} H_{r}^{2} h^{2}}{\rho_{0} \kappa^{2}} .
\end{gathered}
$$

Since now primes will not be written (old variables symbols will be used) but it is important to remember that they are still there. The dimensionless form of Navier-Stokes (11) and thermal diffusion (12) equations are as follows:

$$
\frac{\partial \mathbf{v}}{\partial t}+\mathbf{v} \cdot \nabla \mathbf{v}=-\nabla p+R a \operatorname{Pr}\left(\mathrm{T}-\frac{\mathrm{T}_{0}}{\delta T}\right) \mathbf{k}+\operatorname{Pr} \nabla \cdot \mathbf{S}+M n \mathbf{f}
$$

and

$$
\frac{\partial T}{\partial t}+\mathbf{v} \cdot \nabla T=\nabla^{2} T+\operatorname{Pr} \operatorname{Ec} \eta \Phi+\operatorname{Mn} \operatorname{Ec} T \frac{\partial\left(\chi_{m} \mathbf{H}\right)}{\partial T} \cdot((\mathbf{v} \cdot \nabla) \mathbf{H})
$$

where

$$
\mathbf{f}=\frac{1}{2} \chi_{m}\left(1+\chi_{m}\right) \nabla H^{2}+\chi_{m} \mathbf{H}\left((\mathbf{H} \cdot \nabla) \chi_{m}\right)
$$

and

$$
\chi_{m}=\chi_{m}(T(\mathbf{x}))=\frac{\chi_{0}}{1+(\alpha \delta T)\left(T(\mathbf{x})-\frac{T_{0}}{\delta T}\right)}
$$

Dimensionless Brinkman equations are as follows

$$
\frac{\partial \mathbf{v}_{B}}{\partial t}=-\nabla p_{B}+\operatorname{Pr} \nabla \cdot \mathbf{S}_{B}+\operatorname{Pr} \operatorname{Da} \mathbf{v}_{B} .
$$

In the presents of magnetic field Kelvin body force is added

$$
\frac{\partial \mathbf{v}_{B}}{\partial t}=-\nabla p_{B}+\operatorname{Pr} \nabla \cdot \mathbf{S}_{B}+\operatorname{Pr} \operatorname{Da} \mathbf{v}_{B}+M n \mathbf{f} .
$$

\section{Numerical results}

In this section we present numerical simulation results of heat transfer in ferrofluid. The flow takes place in channel and in channel with porous walls. The two-dimensional time 
dependent flows are assumed viscous, incompressible and laminar. Above the channel magnetic dipole is located. The fluid is assumed to be electrically nonconducting. It is assumed also that there is no electric field effects. This magneto-thermo-mechanical problem is governed by dimensionless equations (17-22).

\subsection{Heat transfer in ferrofluid in channel}

Considered flow takes place in channel between two parallel flat plates. The length of the channel is $L$ and distance between plates is $h$.

The corresponding boundary conditions for dimensionless variables are assumed:

- For the upper wall: the upper wall temperature is kept at constant temperature $T_{u} / \delta T$. The velocity is 0 (no slip condition).

- For the lower wall: the lower wall temperature is kept at constant temperature $T_{l} / \delta T$. The velocity is 0 (no slip condition).

- $\quad$ For inlet (the left wall): the temperature is varying linearly from $T_{l} / \delta T$ to $T_{u} / \delta T$ and is given by equation $T_{i n}=\frac{T_{u}-T_{l}}{\delta T} y+\frac{T_{l}}{\delta T}$ where $\delta T=\left|T_{u}-T_{l}\right|$. There is a parabolic laminar flow profile given by equation $u_{\text {in }}=-4 \frac{u_{0}}{u_{r}} y(y-1)$ for $y \in\langle 0,1\rangle$ at the inlet end.

- For outlet (the right wall) $(x=L, 0 \leq y \leq 1)$ : the convective flux is assumed for temperature, $\mathbf{n} \cdot(-k \nabla T)=0$. Pressure outlet is also assumed, $(-p \mathbf{I}+\mathbf{S}) \mathbf{n}=-p_{0} \mathbf{n}$, where $p_{0}$ is the dimensionless atmospheric pressure.

The following initial conditions for dimensionless variables are assumed: the fluid is motionless, the pressure is zero and the temperature is varying linearly from lower to upper wall.

The time-dependent flow is considered for dimensionless time $t \in\langle 0,0.5\rangle$. The problem is solved with COMSOL code using direct UMFPACK linear system solver. Relative and absolute tolerance used in calculations are 0.05 and 0.005 , respectively.

The following values of temperatures are assumed $T_{l}=T_{0}, T_{u}=T_{0}+\delta T$ where $T_{0}=300 \mathrm{~K}$ and $\delta T=30 \mathrm{~K}$.

\begin{tabular}{|l|l|l|l|}
\hline Quantity & Flow A & Flow B & Flow C \\
\hline$H_{r}$ & $\mathbf{3 8 1 9 . 7 1}$ & $\mathbf{5 0 9 2 . 9 5}$ & $\mathbf{1 2 7 3 2 . 3 9}$ \\
\hline$M n$ & $\mathbf{1 . 6 9 6 1 e + 7}$ & $\mathbf{3 . 0 1 5 4 e + 7}$ & $\mathbf{1 . 8 8 4 6 e + 8}$ \\
\hline$P r$ & 1.4 & 1.4 & 1.4 \\
\hline$R a$ & $2.5701 \mathrm{e}+7$ & $2.5701 \mathrm{e}+7$ & $2.5701 \mathrm{e}+7$ \\
\hline$E c$ & $2.1810 \mathrm{e}-12$ & $2.1810 \mathrm{e}-12$ & $2.1810 \mathrm{e}-12$ \\
\hline$R e$ & 0.7142 & 0.7142 & 0.7142 \\
\hline
\end{tabular}

Table 1. The quatntities for ferrofluid flows A-C

It can be observed that the maximum value of the magnitude of the velocity field of the flow in the channel under the magnetic dipole increases due to the value of the magnetic number. 
a

Time $=0.01$ Surface: Velocity field Contour: Temperature

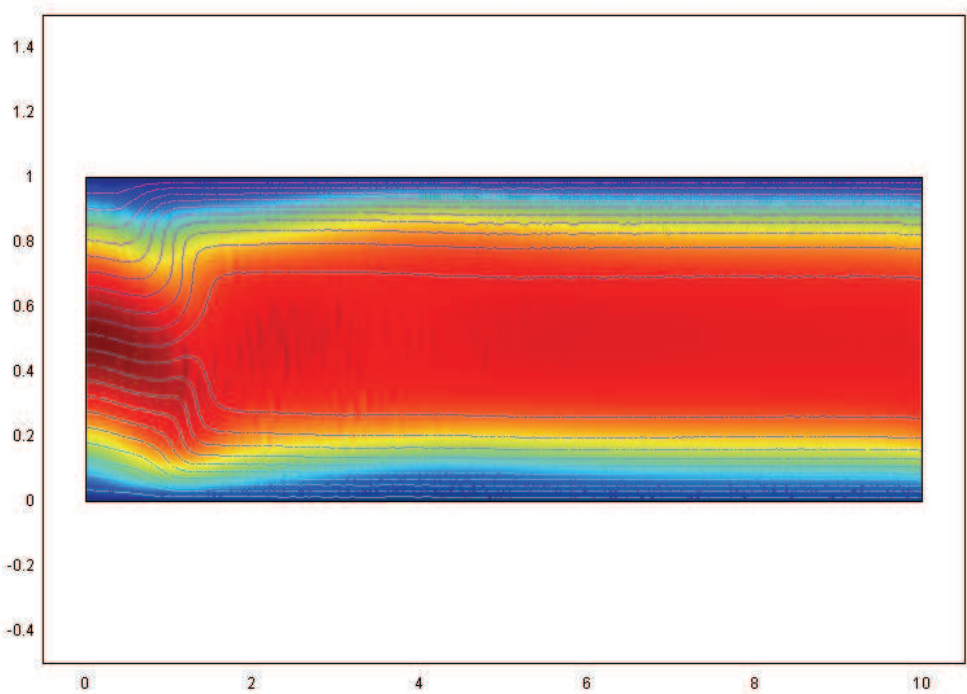

b

Time $=0.3$ Surface: Velocity field Contour: Temperature

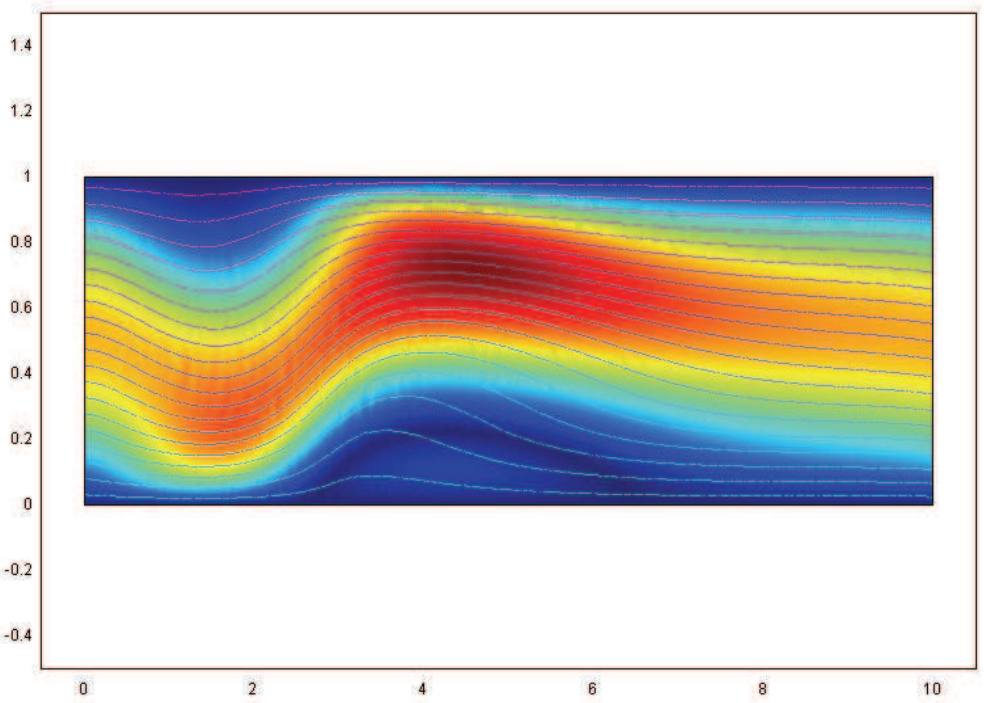

Max. 10.949 Max: 165.402

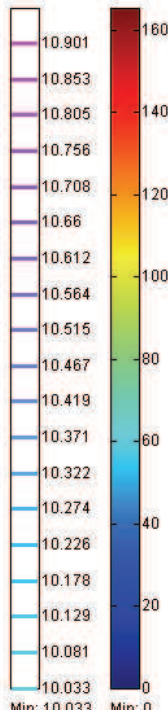

Max. 10.966 Max. 238.995

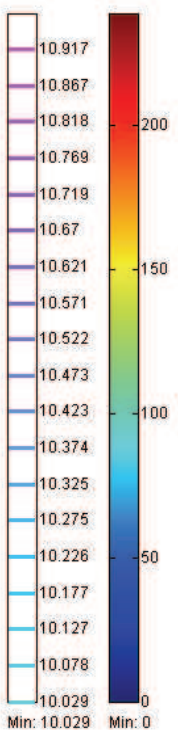

Figure 1. Time evolution of dimensionless velocity field (surface) and temperature contour of flow $B$ for (a) $t=0.01$ (b) $t=0.3$ 
a
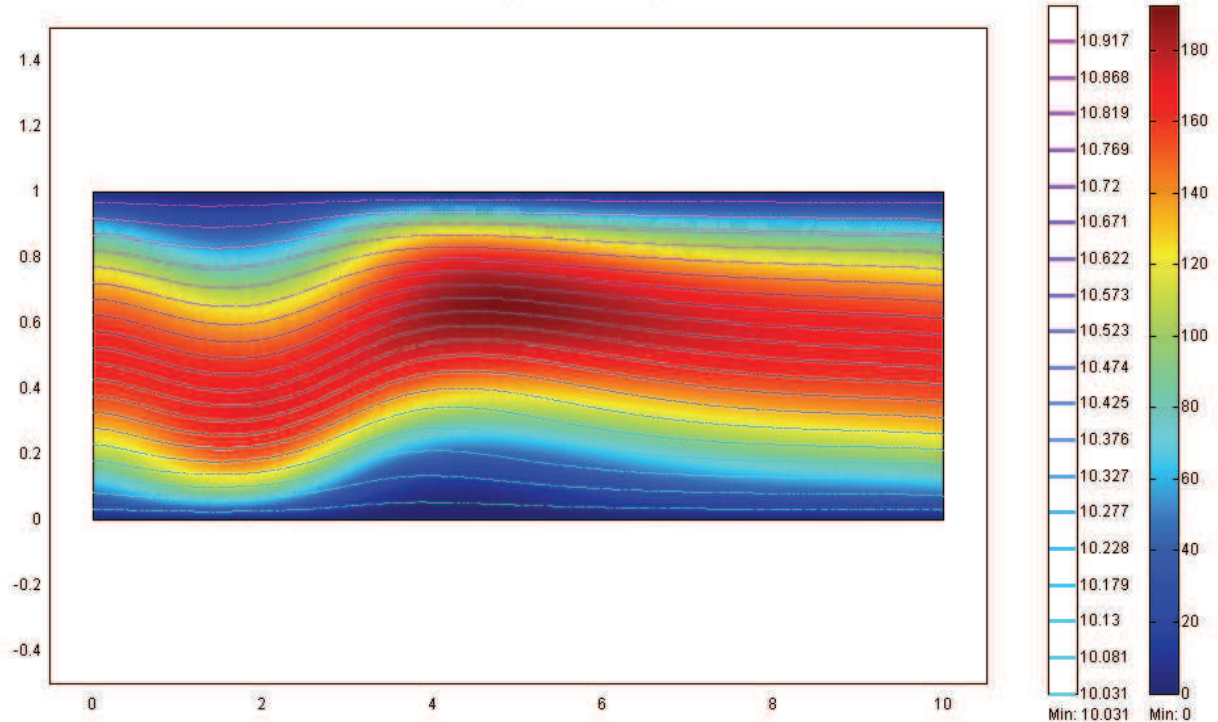

$\mathrm{b}$
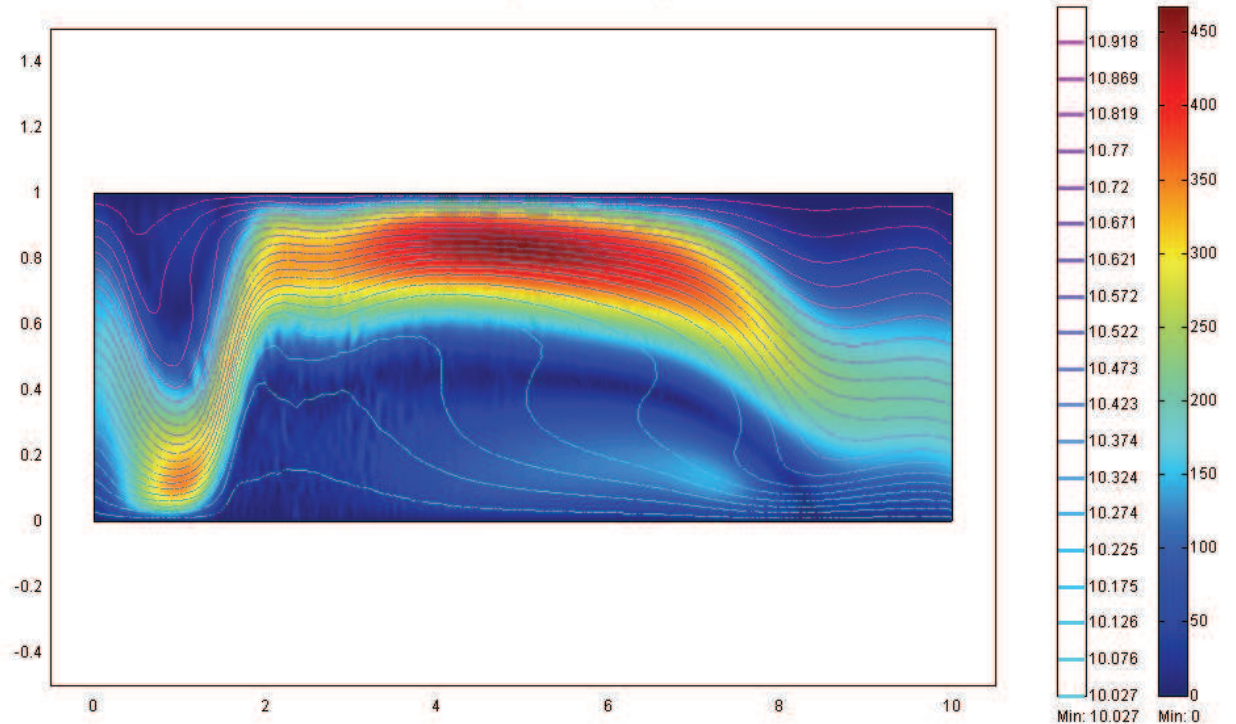

Figure 2. Comparison of dimensionless velocity field (surface) and temperature contour for the different values of magnetic number: (a) flow A (b) flow C 
The flow was relatively uninfluenced by the magnetic field until its strength was large enough for the Kelvin body force to overcome the viscous force. It can be observed that the cooler ferrofluid flows in the direction of the magnetic field gradient and displaced hotter ferrofluid (Fig. 1,2). This effect is similar to natural convection where cooler, more dense material flows towards the source of gravitational force. Ferrofluids have promising potential for the heat transfer applications because a ferrofluid flow can be controlled by using an external magnetic field.

\subsection{Ferroconvection}

Flow takes place in channel between two parallel porous domains. The length of the channel is $L$ and distance between porous domains is $h$. The length of porous domains are $L$ and the height are $4 h / 10$.

The corresponding boundary conditions for dimensionless variables in channel flow are assumed:

- $\quad$ For free-porous structure interface - the upper and the lower wall, free-porous structure interface: $p=p_{B}$. The expression for the pressure at the boundary between the channel and the porous domain states that the pressure is continuous across this interface.

- $\quad$ For inlet (the left wall): the temperature is varying linearly from $T_{l} / \delta T$ to $T_{u} / \delta T$ and is given by equation $T_{i n}=\frac{T_{u}-T_{l}}{\delta T} y+\frac{T_{l}}{\delta T}$ where $\delta T=\left|T_{u}-T_{l}\right|$. There is a parabolic laminar flow profile given by equation $u_{i n}=-4 \frac{u_{0}}{u_{r}} y(y-1)$ for $y \in\langle 0,1\rangle$ at the inlet end.

- For outlet (the right wall): the convective flux is assumed for temperature, $\mathbf{n} \cdot(-k \nabla T)=0$. Pressure outlet is also assumed, $(-p \mathbf{I}+\mathbf{S}) \mathbf{n}=-p_{0} \mathbf{n}$, where $p_{0}$ is the dimensionless atmospheric pressure.

The corresponding boundary conditions for dimensionless variables in porous domain are assumed:

- For free-porous structure interface: $\mathbf{v}_{B}=\mathbf{v}$. These conditions imply that the components of the velocity vector are continuous over the interface between the free channel and the porous domain.

- For the upper domain walls: the temperature is kept at constant temperature $T_{u} / \delta T$. The velocity is 0 (no slip condition).

- For the lower domain walls: the temperature is kept at constant temperature $T_{l} / \delta T$. The velocity is 0 (no slip condition).

The following initial conditions for dimensionless variables are assumed: the fluid is motionless, the pressure is zero and the temperature is $T_{l} / \delta T$.

The time-dependent flow is considered for dimensionless time $t \in\langle 0,0.1\rangle$. The problem is solved with COMSOL code using direct UMFPACK linear system solver. Relative and absolute tolerance used in calculations are 0.05 and 0.005 , respectively. 
The following values of temperatures are assumed $T_{l}=T_{0}, \quad T_{u}=T_{0}+\delta T$ where $T_{0}=300 \mathrm{~K}$ and $\delta T=30 \mathrm{~K}$. The numerical values of variables and dimensionless numbers are presented in Table 2.

The heat transfer in ferrofluid flowing in channel with porous walls is considered in four different flows with different magnetic susceptibility, inlet velocity or permeability of the porous structure. The most interesting example of flow we can observe in the last considered flow (flow G). In this case the ferroconvection is observe (Figures 3d,4-5). We observe convection rolls created near the centre of magnetic dipole. Each roll is moving from left to right where the magnetic field intensity is getting smaller. The intensity of magnetic field is plotted on each figure presented in this subsection as contour lines.

\begin{tabular}{|c|c|c|c|c|}
\hline Quantity & Flow D & Flow E & Flow F & Flow G \\
\hline$H_{r}$ & 12732.39 & 12732.39 & 12732.39 & 12732.39 \\
\hline$\chi_{0}$ & 0.06 & 0.06 & 0.01 & 0.01 \\
\hline$u_{0}$ & $5 e-3$ & $5 e-4$ & $5 e-2$ & $5 e-4$ \\
\hline$M n$ & $1.8846 \mathrm{e}+8$ & $1.8846 \mathrm{e}+8$ & $1.8846 \mathrm{e}+8$ & $1.8846 \mathrm{e}+8$ \\
\hline $\mathrm{Pr}$ & 1.4 & 1.4 & 1.4 & 1.4 \\
\hline$R a$ & $2.5701 \mathrm{e}+7$ & $2.5701 \mathrm{e}+7$ & $2.5701 \mathrm{e}+7$ & $2.5701 \mathrm{e}+7$ \\
\hline Ec & 2.1810e-12 & 2.1810e-12 & $2.1810 \mathrm{e}-12$ & $2.1810 \mathrm{e}-12$ \\
\hline$R e$ & 0.7142 & 0.7142 & 0.7142 & 0.7142 \\
\hline$D a$ & 4000 & 4000 & 40 & 40 \\
\hline
\end{tabular}

Table 2. The quantities for the ferrofluid flows D-G 
a

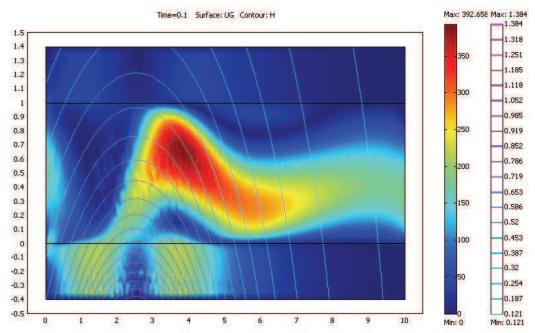

b

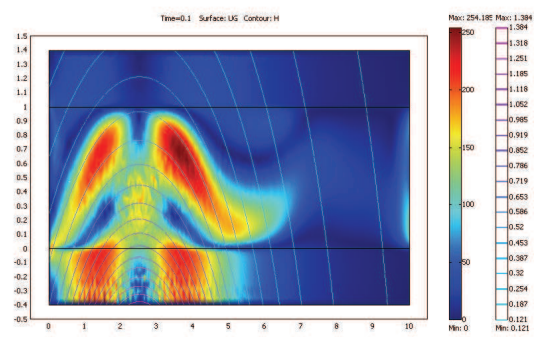

C

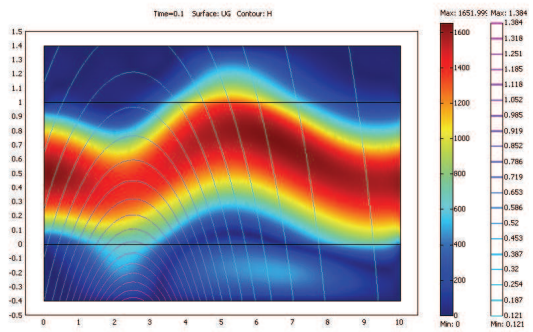

d

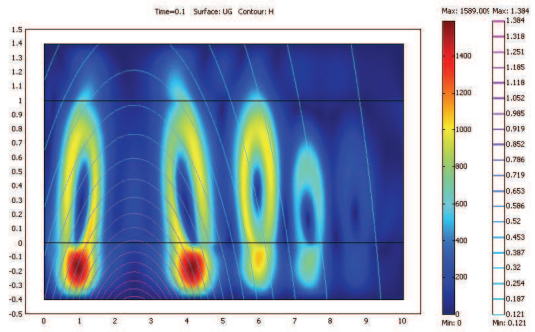

Figure 3. Comparison of dimensionless velocity field for the different flows in channel with porous walls: (a) flow $D(b)$ flow $E(c)$ flow $F(d)$ flow $G$ for time $t=0.1$ 
a
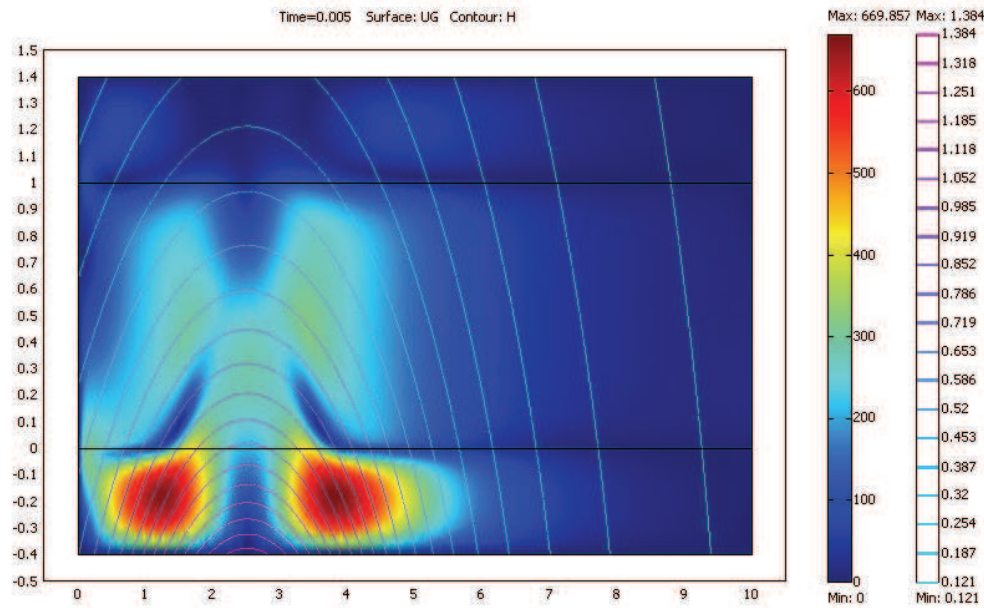

$\mathrm{b}$
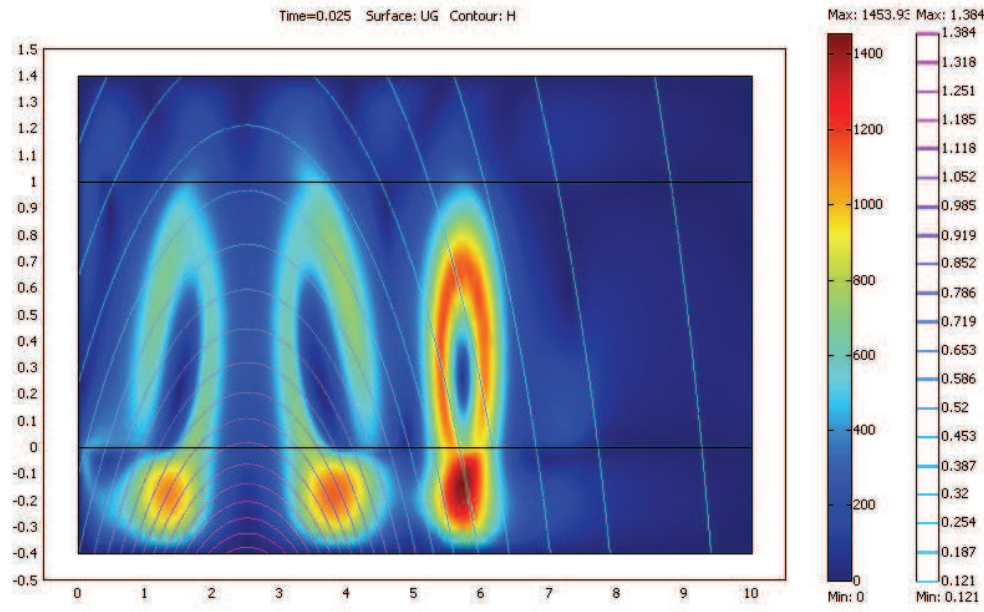

Figure 4. Time evolution of dimensionless velocity field (surface) of flow $G$ for (a) $t=0.005$ (b) $\mathrm{t}=0.025$ 
a
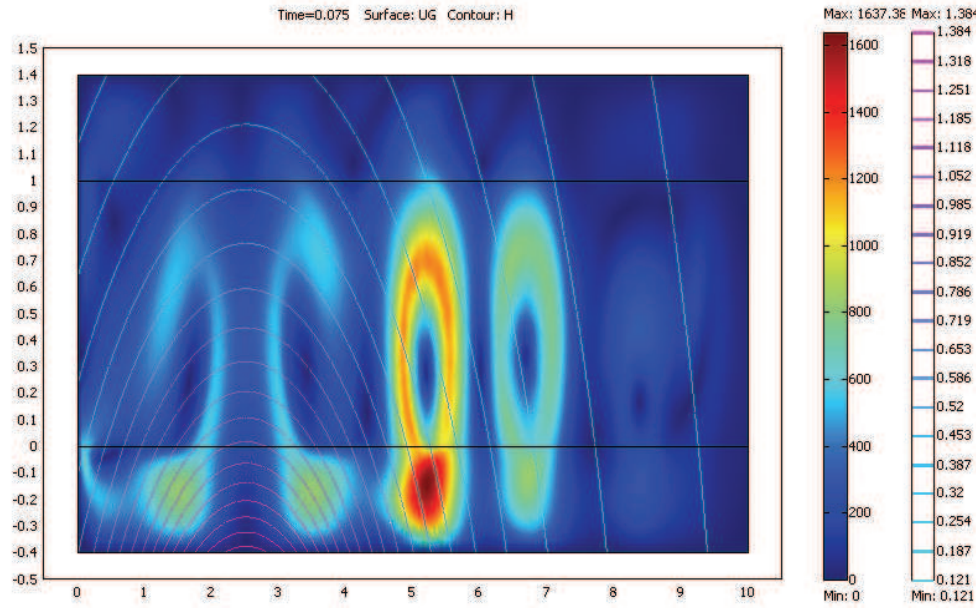

$\mathrm{b}$
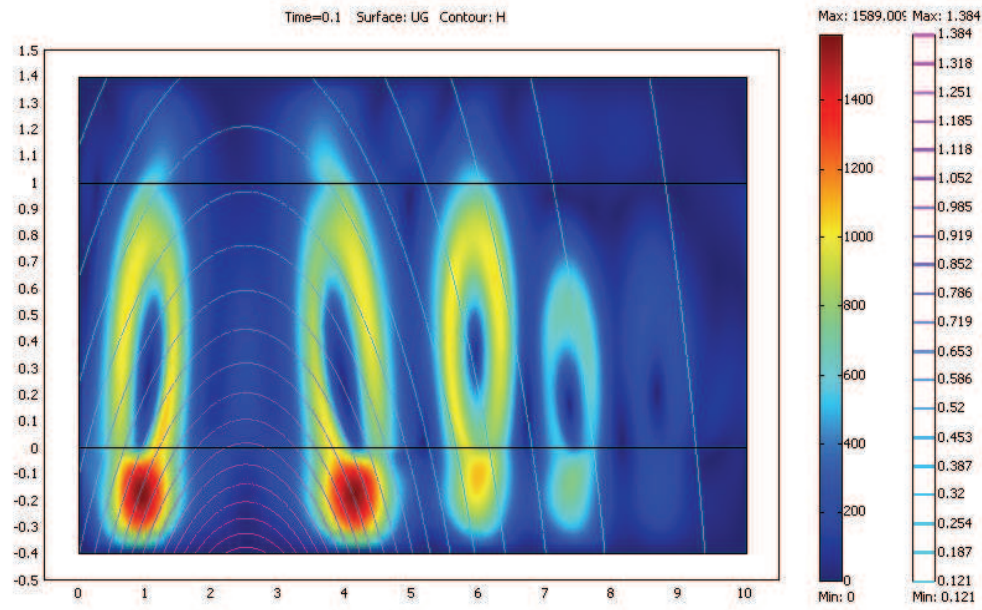

Figure 5. Time evolution of dimensionless velocity field (surface) of flow $G$ for (a) $t=0.075$ (b) $\mathrm{t}=0.1$ 


\subsection{Ferrofluid cooling}

This case examines the temperature field in the ferrofluid and in the electronic component with heat source. The ferrofluid transports heat by convection and conduction. Finally, to approximates the electronic component that requires cooling, the model uses a rectangular blocks with a given volume heat source. The electronic components transports thermal energy by pure conduction.

The viscous, two-dimensional, incompressible and laminar ferromagnetic fluid flow is considered in this subsection. Flow takes place in channel between two parallel flat plates. There are rectangular blocks (heat-generating devices) below the upper wall. The length of the channel is $L=10$ and distance between plates is $h=1$. Outside the channel the magnetic dipole is located at point $(a, b)$.

Good way to describe the convective heat transfer is to model the heat transfer in combination with the fluid-flow field. The results then accurately describe the heat transport and temperature changes. These types of models are somewhat complex but are useful for unusual geometries and complex systems such as circuit-board cooling.

The following boundary conditions for dimensionless variables are assumed:

- For upper wall: The velocity is 0 (no slip condition). Insulation condition for heat transfer by conduction (in solid domain) $\mathbf{n} \cdot \mathbf{q}=\mathbf{n} \cdot\left(-k_{s} \nabla T\right)=0$ and for heat transfer by conduction and convection (in fluid domain) $\mathbf{n} \cdot \mathbf{q}=\mathbf{n} \cdot\left(-k_{f} \nabla T+\rho_{f} c_{f} T \mathbf{u}\right)=0$ specifies where the domain is well insulated.

- For lower wall: The velocity is 0 (no slip condition). Insulation condition for heat transfer by conduction and convection (in fluid domain) $\mathbf{n} \cdot \mathbf{q}=\mathbf{n} \cdot\left(-k_{f} \nabla T+\rho_{f} \mathcal{c}_{f} T \mathbf{u}\right)=0$.

- For inlet (left wall): The temperature is $\frac{T_{l}}{\delta T}$ where $\delta T=\left|T_{u}-T_{l}\right|$. At the inlet boundary there is a parabolic laminar flow profile given by equation $u_{\text {in }}=-4 \frac{u_{0}}{u_{r}} y(y-1)$ for $y \in\langle 0,1\rangle$.

- For outlet (right wall): The convective flux is assumed for temperature, $\mathbf{n} \cdot\left(-k_{s} \nabla T\right)=0$. Pressure outlet is also assumed, $(-p \mathbf{I}+\mathbf{S}) \mathbf{n}=-p_{0} \mathbf{n}$, where $p_{0}$ is the dimensionless atmospheric pressure.

- For solid-fluid interface: The velocity is 0 (no slip condition). Continuity equation for heat transfer equation $\mathbf{n} \cdot\left(\mathbf{q}_{s}-\mathbf{q}_{f}\right)=0 \quad$ where $\mathbf{q}_{s}=-k_{s} \nabla T \quad$ and $\mathbf{q}_{f}=-k_{f} \nabla T+\rho_{f} c_{f} T \mathbf{u}$.

The following initial conditions for dimensionless variables are assumed: fluid is motionless (velocity is zero), pressure is zero and temperature is $\frac{T_{l}}{\delta T}$ for whole domain (with fluid and solid).

Numerical results of solved problem of ferrofluid cooling of heat-generating device in channel flow under the influence of magnetic dipole generated by permanent magnet are presented below. The numerical values of variables and dimensionless numbers are 
presented in Table 3 and Table 4. Problem was solved using finite element method with 2373 Lagrange quadratic triangular mesh elements and 15242 degrees of freedom. Timedependent flow is considered for time $t \in\langle 0,0.5\rangle$. The problem is solved with finite element method COMSOL code using direct UMFPACK linear system solver. Relative and absolute tolerance used in calculations are 0.05 and 0.005 , respectively.

\begin{tabular}{|c|c|c|c|c|}
\hline Quantity & Flow $\mathrm{H}$ & Flow I & Flow J & Flow K \\
\hline $\begin{array}{l}a \\
b\end{array}$ & $\begin{array}{c}2 \\
-3\end{array}$ & $\begin{array}{c}2 \\
-2.5\end{array}$ & $\begin{array}{c}2 \\
-2\end{array}$ & $\begin{array}{c}2 \\
-1.5\end{array}$ \\
\hline$H_{r}$ & $1.768388 \mathrm{e}+5$ & $2.546479 \mathrm{e}+5$ & $3.978874 \mathrm{e} 5$ & $7.073553 e+5$ \\
\hline $\operatorname{Pr}$ & \multicolumn{4}{|c|}{560} \\
\hline$R a$ & \multicolumn{4}{|c|}{200.7938} \\
\hline$E c$ & \multicolumn{4}{|c|}{$1.16324 \mathrm{e}-13$} \\
\hline$R e$ & \multicolumn{4}{|c|}{0.001786} \\
\hline$M n$ & $2.272182 \mathrm{e}+9$ & $4.711597 \mathrm{e}+9$ & $1.150292 \mathrm{e}+10$ & $3.635492 \mathrm{e}+10$ \\
\hline$Q n$ & \multicolumn{4}{|c|}{64.019097} \\
\hline$V_{a v g}$ (fluid) & 292.980369 & 309.281869 & 331.905089 & 371.38104 \\
\hline$T_{a v g}$ (fluid) & 101.330192 & 101.314674 & 101.096441 & 100.801837 \\
\hline$T_{\text {dom (channel) }}$ & 118.521428 & 118.261882 & 117.760089 & 117.17941 \\
\hline
\end{tabular}

Table 3. Quantities of flow H-K

\begin{tabular}{|c|c|c|c|c|}
\hline Quantity & Flow L & Flow M & Flow N & Flow O \\
\hline$\chi_{0}$ & 0.5 & 0.1 & 0.06 & 0.04 \\
\hline$H_{r}$ & \multicolumn{4}{|c|}{$1.768388 \mathrm{e}+5$} \\
\hline $\operatorname{Pr}$ & \multicolumn{4}{|c|}{560} \\
\hline$R a$ & \multicolumn{4}{|c|}{200.7938} \\
\hline$E c$ & \multicolumn{4}{|c|}{$1.16324 \mathrm{e}-13$} \\
\hline$R e$ & \multicolumn{4}{|c|}{0.001786} \\
\hline$M n$ & \multicolumn{4}{|c|}{$2.272182 \mathrm{e}+9$} \\
\hline$Q n$ & \multicolumn{4}{|c|}{64.019097} \\
\hline$V_{a v g}$ (fluid) & 411.490459 & 306.564373 & 292.980369 & 284.589693 \\
\hline$T_{a v g}$ (fluid) & 100.741705 & 101.246393 & 101.330192 & 101.453763 \\
\hline$T_{\text {dom (channel) }}$ & 116.618815 & 118.171175 & 118.521428 & 118.846783 \\
\hline
\end{tabular}

Table 4. Quantities of flow L-O 
a

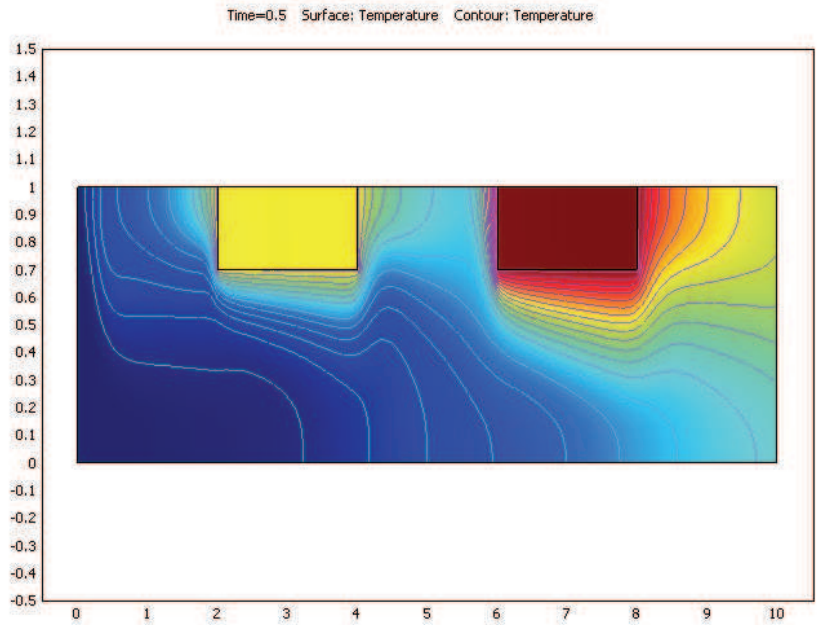

Max: 15.298 Max: 15.165

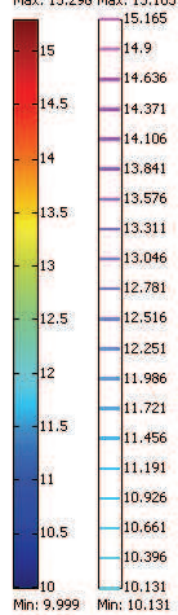

$\mathrm{b}$
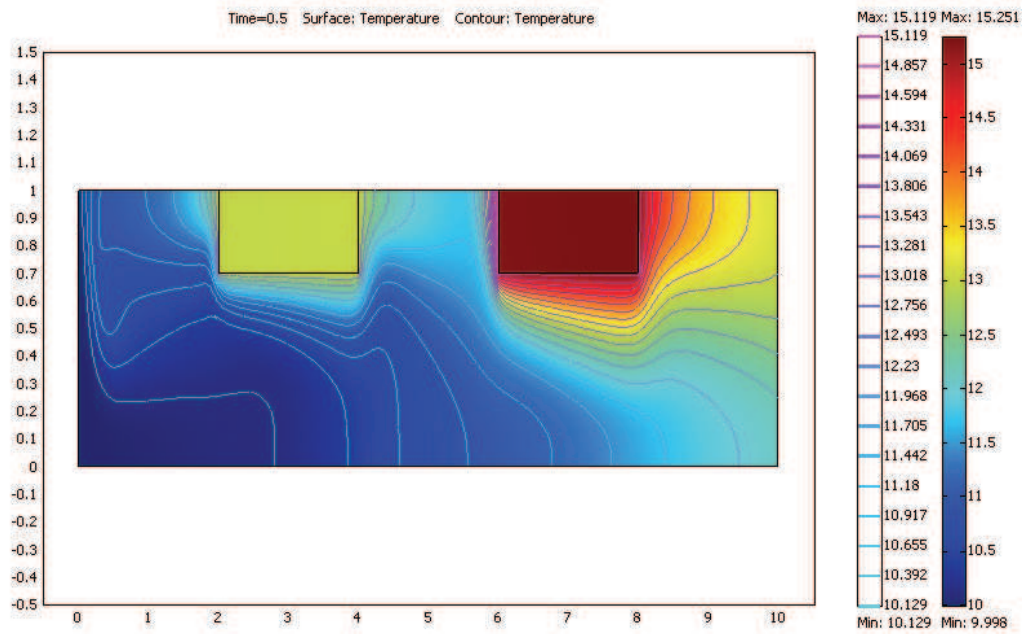

Figure 6. Comparison of temperature in channel and heat generating devices for the different flows in channel: (a) flow $\mathrm{H}$, (b) flow I for time $t=0.5$ 
a
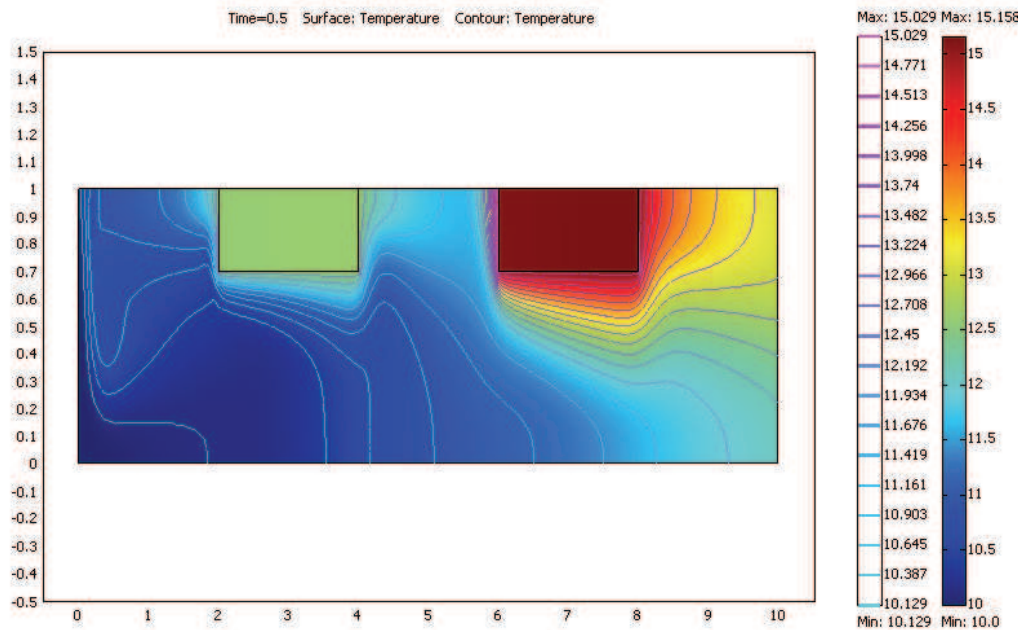

$\mathrm{b}$
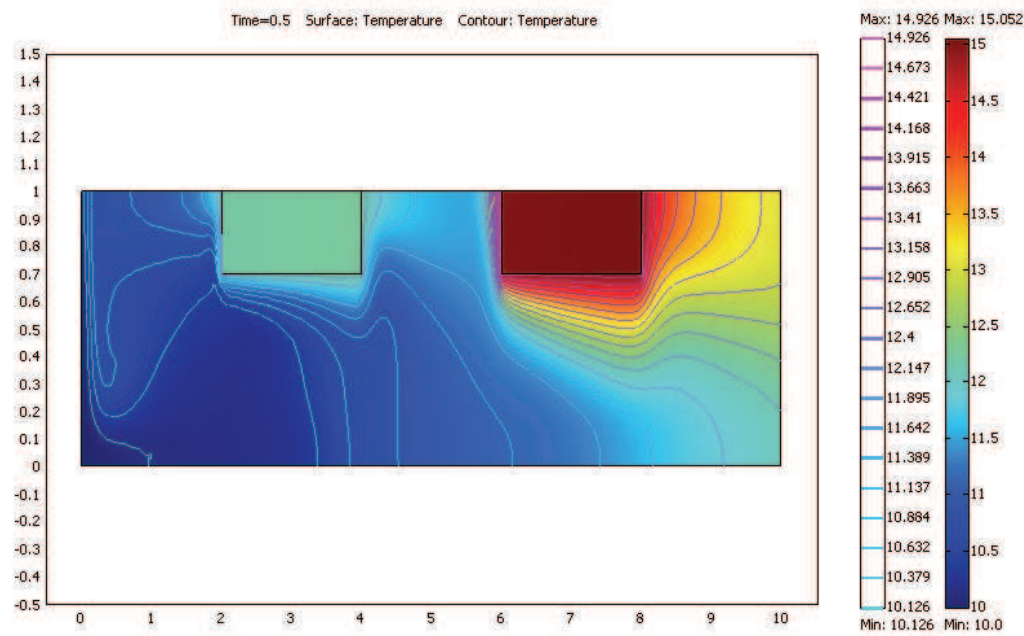

Figure 7. Comparison of temperature in channel and heat generating devices for the different flows in channel: (a) flow J, (b) flow $\mathrm{K}$ for time $\mathrm{t}=0.5$ 
a
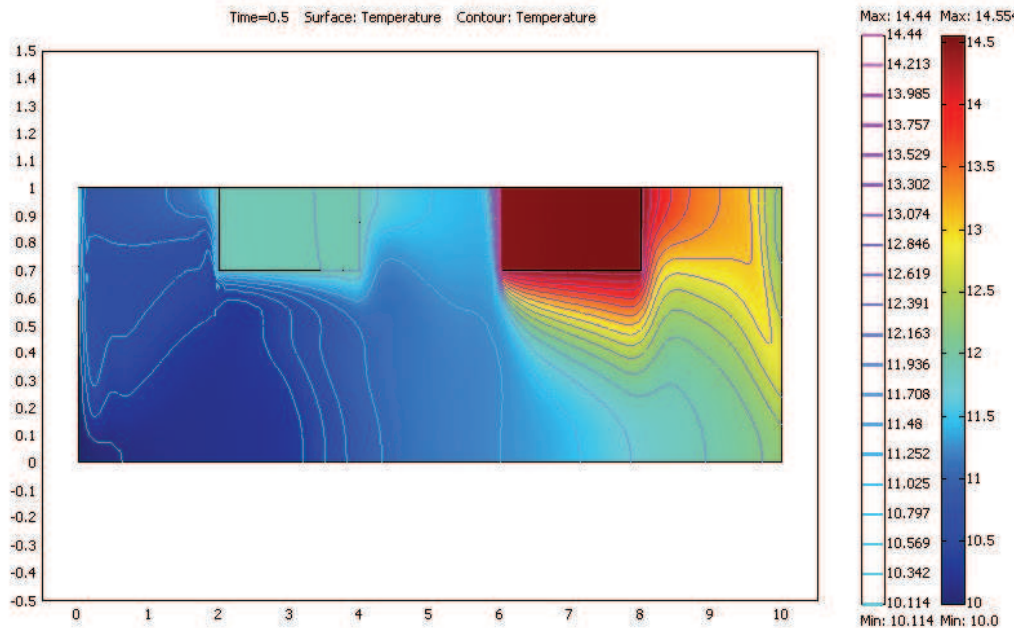

$\mathrm{b}$
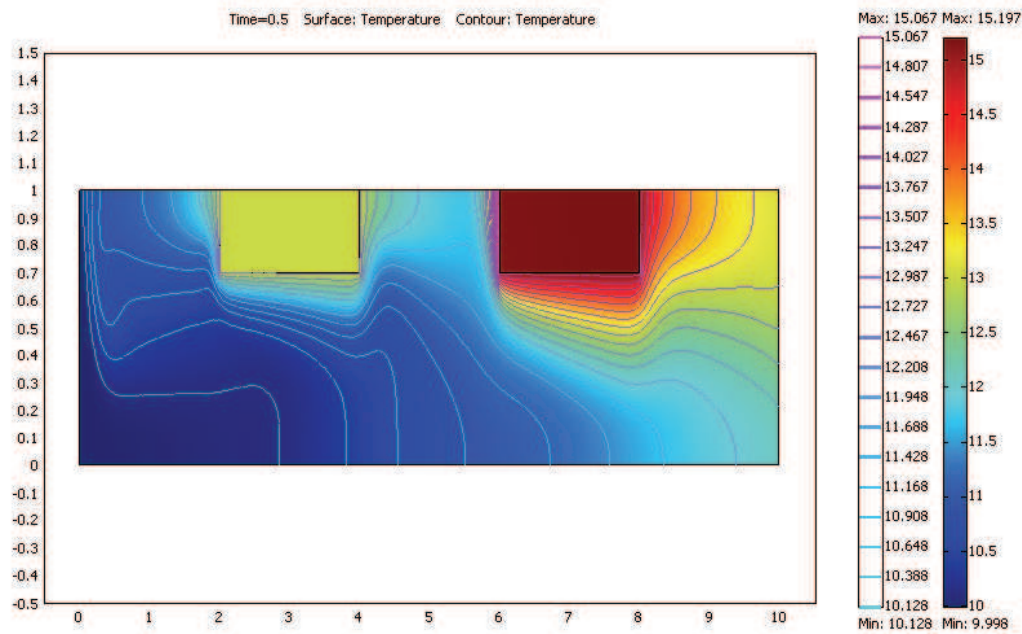

Figure 8. Comparison of temperature in channel and heat generating devices for the different flows in channel: (a) flow $\mathrm{L}$, (b) flow $\mathrm{M}$ for time $\mathrm{t}=0.5$ 
a
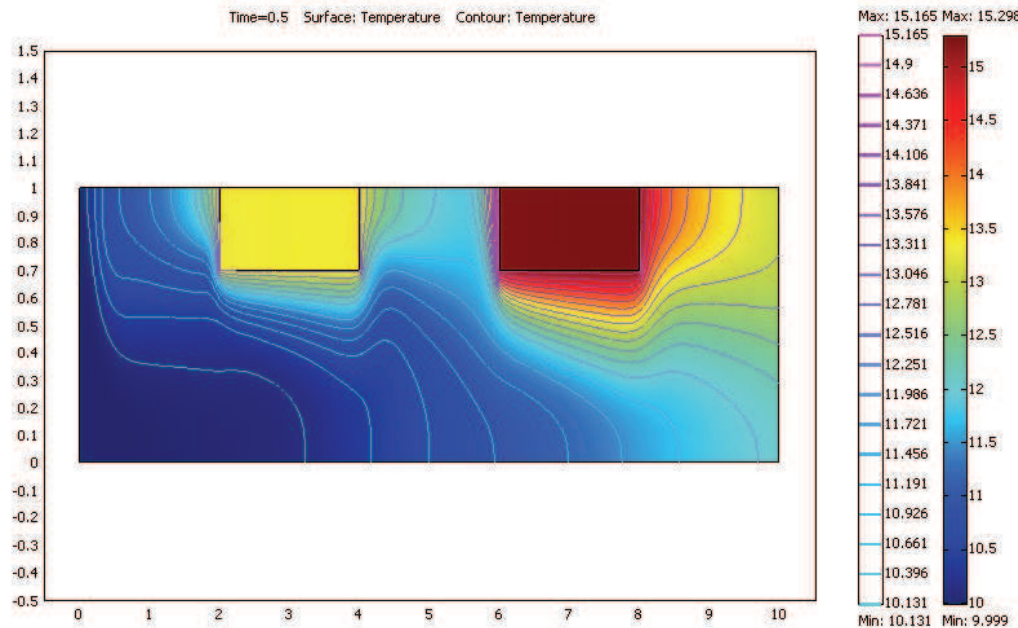

b
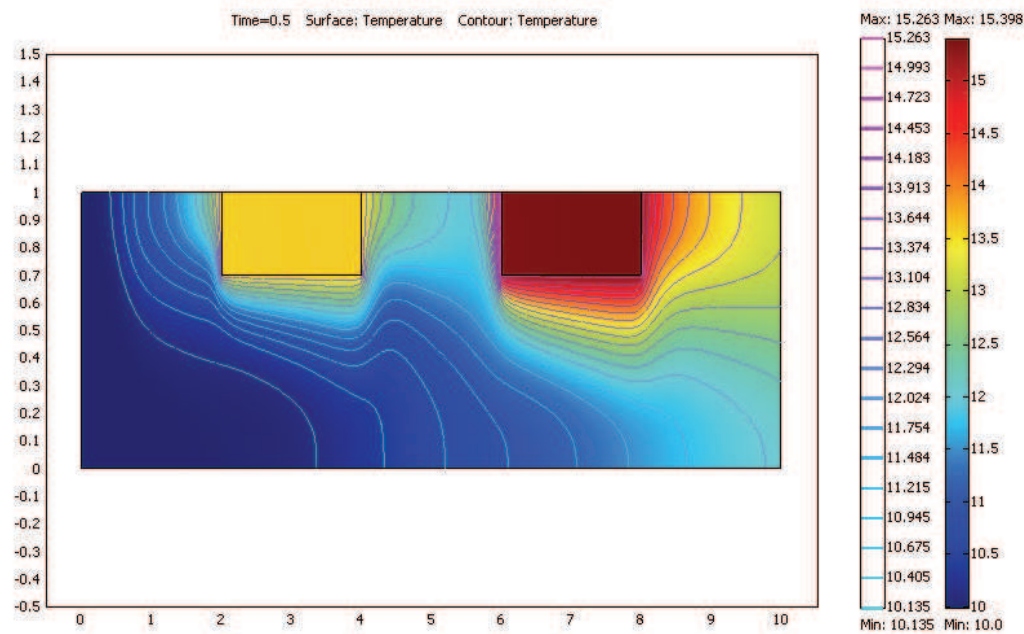

Figure 9. Comparison of temperature in channel and heat generating devices for the different flows in channel: (a) flow $\mathrm{N}$, (b) flow $\mathrm{O}$ for time $\mathrm{t}=0.5$ 
Dimensionless values $V_{a v g}, T_{a v g}$ and $T_{\text {dom }}$ are calculated using following formulas

$$
\begin{aligned}
V_{a v g} & =\iint_{A_{p}}|\mathbf{u}| d A_{p}, \\
T_{a v g} & =\iint_{A_{p}} T d A_{p}, \\
T_{d o m} & =\iint_{A} T d A,
\end{aligned}
$$

where $A_{p}$ denotes area of domain with fluid, $A$ - area of the channel (fluid and solid). In these examples we have $A_{p}=8.8$ and $A=10$.

\section{Conclusions}

We have simulated two-dimensional heat transfer in ferrofluid channel flow under the influence of the magnetic field created by magnetic dipole using computational fluid dynamics code COMSOL based on finite element method. At the left end of rectangular channel there was assumed a parabolic laminar flow profile. The upper plate was kept at constant temperature $T_{u}$ and the lower at $T_{l}$.

The flow was relatively uninfluenced by the magnetic field until its strength was large enough for the Kelvin body force to overcome the viscous force. The magnetoconvection was induced by the presence of magnetic field gradient.

We observed that the cooler ferrofluid flows in the direction of the magnetic field gradient and displaced hotter ferrofluid. Ferrofluids have promising potential for heat transfer applications because a ferrofluid flow can be controlled by using an external magnetic field. The Kelvin body force arises from the interaction between the local magnetic field within the ferrofluid and the molecular magnetic moments characterized by the magnetization. An imposed thermal gradient produces a spatial variation in the magnetization through the temperature-dependent magnetic susceptibility for ferrofluids and therefore renders the Kelvin body force non-uniform spatially. This thermal gradient induced inhomogeneous magnetic body force can promote or inhibit convection in a manner similar to the gravitational body force.

A strong magnet placed near the device which produces heat will always attract colder ferrofluid towards it more than warmer ferrofluid thus forcing the heated ferrofluid away, towards the heat sink. This is an efficient cooling method which requires no additional energy input.

\section{References}

Ganguly R., Sen S., Puri I.K (2004). Heat transfer augmentation using a magnetic fluid under the influence of a line dipole, Journal of Magnetism and Magnetic Materials, 271 (2004) pp.63-73. 
Ramanathan A., Suresh G. (2004). Effect of magnetic field dependent viscosity and anisotropy of porous medium on ferroconvection, International Journal of Engineering Science, 42, pp.411-425.

Ritter J.A., Ebner A.D., Daniel K.D, Stewart K.L (2004). Application of high gradient magnetic separation principles to magnetic drug targeting, Journal of Magnetism and Magnetic Materials, 280, s.184-201.

Rosensweig R.E. (1985). Ferrohydrodynamics, Cambridge University Press, Cambridge.

Snyder S.M., Cader T., Finlayson B.A. (2003). Finite element model of magnetoconvection of a ferrofluid, Journal of Magnetism and Magnetic Materials, 262, pp.269-279.

Strek T. (2005). Ferrofluid channel flow under the influence of magnetic dipole, International Journal of Applied Mechanics and Engineering, vol. 10, pp. 103-109.

Strek T., Jopek H. (2007). Computer simulation of heat transfer through a ferrofluid, Physica Status Solidi (B), 244, No. 3, pp. 1027-1037.

Tangthieng C., Finlayson B.A., Maulbetsch J., Cader T. (1999). Heat transfer enhancement in ferrofluids subjected to steady magnetic fields, Journal of Magnetism and Magnetic Materials, 201, pp.252-255.

Yamaguchi H., Zhang Z., Shuchi S., Shimada K. (2002). Heat transfer characteristic of magnetic fluid in a partitioned rectangular box, Journal of Magnetism and Magnetic Materials, 252, pp.203-205.

Voltairas P.A., Fotiadis D.I., Michalis L.K. (2002). Hydrodynamics of magnetic drug targeting, Journal of Biomechanics, 35, pp. 813-821. 


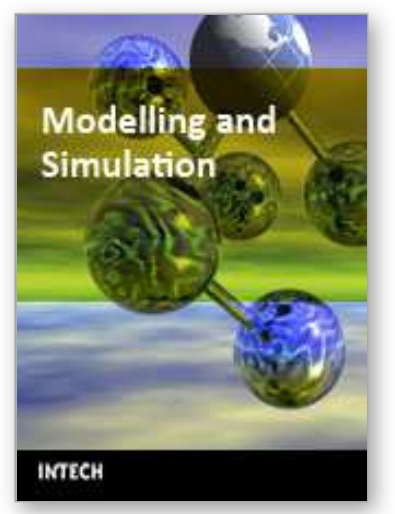

\author{
Modelling and Simulation \\ Edited by Giuseppe Petrone and Giuliano Cammarata
}

ISBN 978-3-902613-25-7

Hard cover, 688 pages

Publisher I-Tech Education and Publishing

Published online 01, June, 2008

Published in print edition June, 2008

This book collects original and innovative research studies concerning modeling and simulation of physical systems in a very wide range of applications, encompassing micro-electro-mechanical systems, measurement instrumentations, catalytic reactors, biomechanical applications, biological and chemical sensors, magnetosensitive materials, silicon photonic devices, electronic devices, optical fibers, electro-microfluidic systems, composite materials, fuel cells, indoor air-conditioning systems, active magnetic levitation systems and more. Some of the most recent numerical techniques, as well as some of the software among the most accurate and sophisticated in treating complex systems, are applied in order to exhaustively contribute in knowledge advances.

\title{
How to reference
}

In order to correctly reference this scholarly work, feel free to copy and paste the following:

Tomasz Strek (2008). Finite Element Simulation of Heat Transfer in Ferrofluid, Modelling and Simulation, Giuseppe Petrone and Giuliano Cammarata (Ed.), ISBN: 978-3-902613-25-7, InTech, Available from: http://www.intechopen.com/books/modelling_and_simulation/finite_element_simulation_of_heat_transfer_in_fe rrofluid

\section{INTECH}

open science | open minds

\section{InTech Europe}

University Campus STeP Ri Slavka Krautzeka 83/A 51000 Rijeka, Croatia Phone: +385 (51) 770447 Fax: +385 (51) 686166 www.intechopen.com

\section{InTech China}

Unit 405, Office Block, Hotel Equatorial Shanghai

No.65, Yan An Road (West), Shanghai, 200040, China

中国上海市延安西路65号上海国际贵都大饭店办公楼405单元

Phone: +86-21-62489820

Fax: +86-21-62489821 
(C) 2008 The Author(s). Licensee IntechOpen. This chapter is distributed under the terms of the Creative Commons Attribution-NonCommercialShareAlike-3.0 License, which permits use, distribution and reproduction for non-commercial purposes, provided the original is properly cited and derivative works building on this content are distributed under the same license. 